BMJ Open

Sport \&

Exercise

Medicine

\title{
Motocross biking for competition and for recreation: a prospective analysis of 423 injured riders
}

\author{
Rohit Singh, Peter Theobald, AK Hamad, Stuart Hay
}

To cite: Singh $R$, Theobald $P$, Hamad AK, et al. Motocross biking for competition and for recreation: a prospective analysis of 423 injured riders. BMJ Open Sport Exerc Med 2015:1:e00019.

doi:10.1136/bmjsem-2015000019

- Prepublication history for this paper is available online To view these files please visit the journal online (http://dx.doi.org/10.1136/ bmjsem-2015-000019).

Accepted 2 August 2015

\section{CrossMark}

Department of SPR Trauma and Orthopaedics, Royal Shrewsbury Hospital, Robert Jones Agnes Hunt Orthopaedic Hospital and Cardiff University, UK

Correspondence to Rohit Singh;

rohitamolsingh@hotmail.com

\section{ABSTRACT}

Background: Motocross is a form of motorcycle racing held on established off-road circuits and has been a recreational and competitive sport across the world for over 100 years. In the UK alone, motocross has grown into a phenomenally ambitious and popular franchise. There are over 200 motocross clubs across the country, permitting over 900 events annually. Method: Data were collected prospectively over 4 years (from 2010 to 2014) at our unit. All injuries caused by motocross biking that were referred to our trauma and orthopaedic department were included in this study, regardless of whether the rider was performing the sport competitively or recreationally.

Results: During the period studied (4 years), 423 patients were included with a total of 485 injuries, ranging from 1 to 6 injuries per patient. The patient's age range was from 4 to 73 years, with most of the injuries being sustained within the early spring and summer months representing the start of the motocross season. Conclusions: We present the first epidemiological study of motocross injuries in the UK. Within the growing culture of 'adrenaline sports', motocross has become an exhilarating and extremely fashionable pastime. This study has identified and categorised the spectrum of injuries from upper limb fracture dislocations to lifethreatening head and chest injuries, some of which are serious and may cause significant morbidity and possible mortality. These injuries could have significant resource implications, especially for smaller rural hospitals.

\section{BACKGROUND}

Motocross is a form of motorcycle racing held on established off-road circuits and has been a recreational and competitive sport across the world for over 100 years. It initially evolved from motorcycle trials competitions back in 1906. ${ }^{12}$ The original term for motocross was described as 'scrambling', said to have originated in the phrase 'a rare old scramble' describing one such early race. ${ }^{1}$ As the sport grew in popularity across the globe, the international competitions became more commonly known as motocross racing, by combining the French word for motorcycle, motocyclette, or moto for short, into a portmanteau with 'cross country'. 2 The first

\section{What are the new findings?}

- This is the first epidemiological study of motocross injuries in the UK of this magnitude.

- The number of motocross injuries has more than doubled from 74 to 170 in the past 4 years.

- Almost half the patients seen with motocross injuries require operative intervention.

- Motocross injuries can be life-threatening.

competitive event in the UK took place at Camberley, Surrey in $1924 .^{3}$

In the UK alone, motocross has grown into a phenomenally ambitious and popular franchise. There are over 200 motocross clubs across the country, permitting over 900 events annually.

The documented age groups for motocross competitive racing are from the age of 6 years for boys and girls to the age of 65 years, for competitive events. However, it has been well documented that children as young as 3 years have taken part competitively. ${ }^{5}$

The majority of events take place between March and October; however, the recreational side of the sport continues throughout the year. ${ }^{4}$ The prerequisites for becoming a competitive motocross rider include the completion of an online license application and the payment of entrance fees totalling approximately 150 pounds; therefore, motocross is seen to be more accessible when compared with other motor vehicle sports. ${ }^{4}$

In 2001, our unit conducted an epidemiological study looking at mountain biking injuries. At that time, the study was revolutionary in highlighting and documenting these injuries for the first time in the UK. ${ }^{6}{ }^{7}$ Interestingly, while much has been previously documented regarding the injuries associated with other forms of competitive motorcycling, a similar documentation of motocross injuries has not been undertaken, despite the sport's obvious popularity. ${ }^{8} 9$ The only other epidemiological study on motocross injuries was carried out in Italy during the years from 


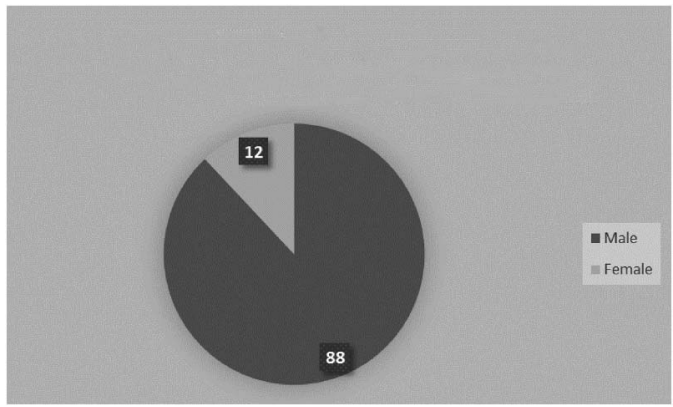

Figure 1 Pie chart showing percentage sex distribution of motocross injuries.

1980 to $1991 .{ }^{10}$ We therefore present the first comprehensive, prospective study of its type in the UK.

\section{METHOD}

Data were collected prospectively over 4 years (from 2010 to 2014) at our unit. All injuries caused by motocross biking that were referred to our trauma and orthopaedic department were included in this study, regardless of whether the rider was performing the sport competitively or recreationally (training). Patients were either seen acutely in the emergency department or subsequently in the fracture clinic.

We specifically looked at motocross riders only; other motor biking injuries and mountain biking injuries were excluded from the study.

Injuries were identified in 423 patients who were directly referred to our department following recreational or competitive motocross.

The information received included basic details (name, age and sex), the type of injury sustained, the need for admission/surgery and any associated complications. The information was prospectively collected from dictated hospital notes (inpatient and outpatient) from trauma and orthopaedic consultants and specialist registrars in our department. Results were tabulated using an Excel spreadsheet (Microsoft, Redmond, Washington).

To facilitate data handling, the injuries were then classified into 16 categories according to the severity and the anatomical region of injury.

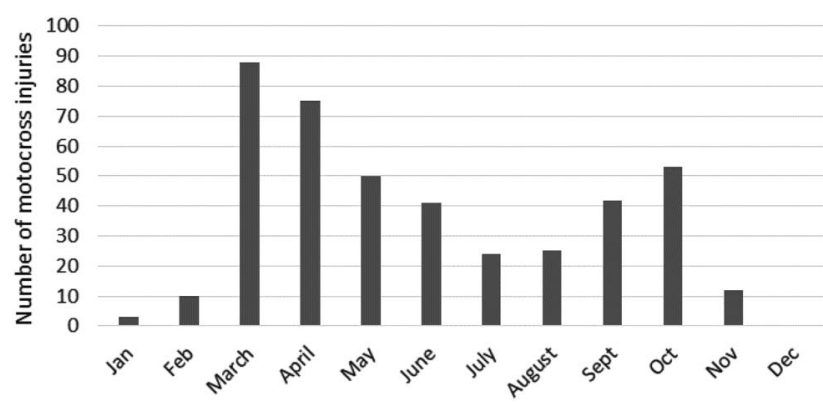

Figure 3 Bar chart showing monthly distribution of motocross injuries.

\section{RESULTS}

During the period studied (4 years), 423 patients were referred to our trauma and orthopaedic department. A total of 485 injuries were recorded, ranging from 1 to 6 injuries per patient. The study period was from August 2010 to August 2014. The vast majority of injuries occurred in patients engaged in the sport for competitive purposes as opposed to training/recreational purposes.

The vast majority of the injuries sustained were in male patients $(\mathrm{n}=372,88 \%$; figure 1$)$.

The patient's age range was from 4 to 73 years, but the main cohort was from the 12 to 54 years of age group, with the majority in the 11-20 years of age group (figure 2).

Most of the injuries were sustained within the early spring and summer months, with the majority in March and April (figure 3). This would represent the start of the motocross season, where riders may be more vulnerable after the winter break, being unaccustomed to the competitive terrain and 'rusty' in terms of skill level. There is a noticeable peak again during the transition between autumn and winter where we suspect the course is more treacherous as the weather and surface conditions become more hazardous.

A total of $205(48 \%)$ patients required operative treatment (figure 4), some needing multiple procedures (2 or more procedures) with a prolonged hospital stay of five of more days $(n=52,12 \%)$.

There was a vast range of injuries with a spectrum ranging from simple soft tissue lacerations through to life-threatening injuries (figure 5).
Figure 2 Bar chart showing age distribution of motocross injuries.

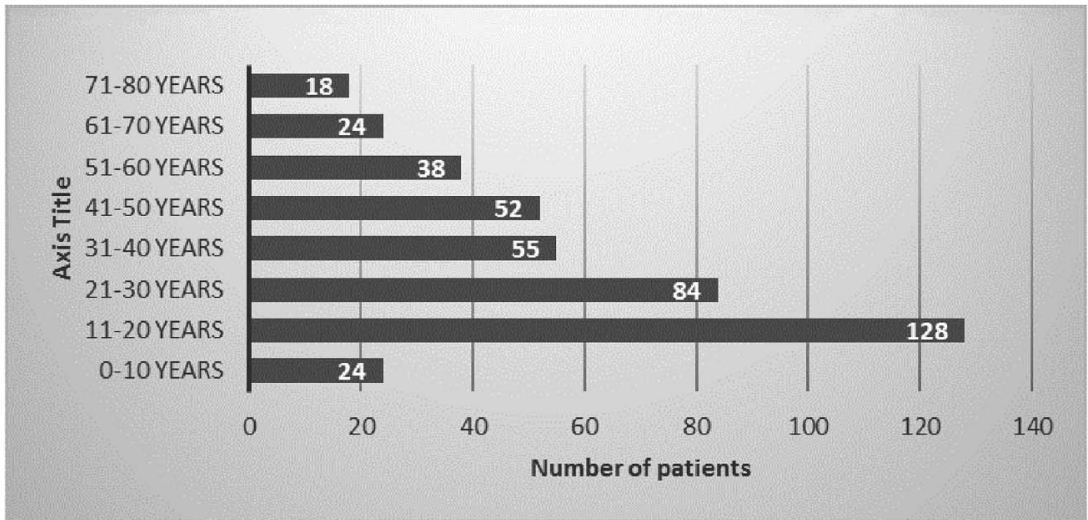




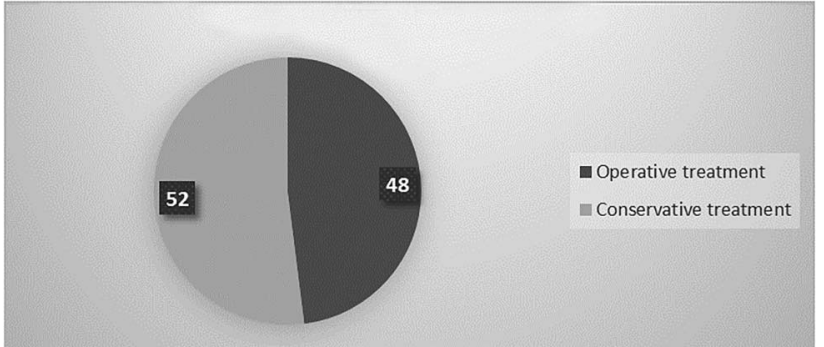

Figure 4 Pie chart showing percentage of motocross patients receiving operative treatment.

The most common injury was clavicular fractures $(\mathrm{n}=70,14 \%)$, followed by long bone fracture $(n=5311 \%)$, forearm/distal radius fracture $(n=47,9.7 \%)$ and other shoulder injuries $(n=43,8.9 \%)$.

A significant proportion of these patients suffered two or more injuries $(n=76,18 \%)$.

In total, 216 patients over the study period required inpatient hospital stay. The length of hospital stay ranged from 1 to 7 days, with the majority staying in hospital for 3 days. Forty patients required admission to high dependency unit/intensive care unit.

All patients required formal orthopaedic follow-up in the fracture clinic. One hundred and fifty-three patients were not resident in the local area of our unit, and therefore the follow-up for these patients was arranged at their respective local departments. All our local patients required at least two follow-up appointments, regardless of whether their treatment was operative or non-operative.

During the 4 years that this study has been conducted, we have seen a steady rise in the number of injuries (figure 6).

In the first year we saw 74 injuries, compared with 170 injuries in the fourth year, showing that the number of injuries has more than doubled in the past 4 years.

\section{DISCUSSION}

This study of motocross injuries represents the first epidemiological study of its type and size in the UK, significant by both by its magnitude and by the variety of injuries incurred.

Competitive and recreational motocross is an increasingly popular sport both in the UK and worldwide, where the number of annual tournaments and racers has doubled in the past 5 years. ${ }^{2}{ }^{3}$ Children as young as 3 years of age have participated in this activity. It is recognised as a high-risk sport despite the use of protective equipment (kidney belts, neck braces, helmets, goggles, knee and elbow supports) and modifications in course design. Those injuries sustained range from minor contusions and lacerations to severe lifethreatening spinal and head injuries both in the adult and the paediatric population.

\section{ON-SITE SAFETY MEASURES}

The surge of trauma generated from these events can cause a significant burden on small local hospitals after large, competitive events despite the safety measures taken at the venue. These safety measures include on-site health and safety management, license applications, local authority liaison, crowd management planning, fire safety management, crisis management planning, safe working practice documents and pedestrian management solutions. ${ }^{4} 11$ However, such events can still result in a significant increase in workload and subsequent delays in treatment. It is interesting to note that $82 \%$ of injuries documented in our study resulted from competition events, which further emphasises the need for raised awareness of these events. With the advent of national trauma centres, there is an argument that the location of these motocross competitions would be preferable to the adjacent vicinity to such centres.

\section{INJURY TYPE AND LEVEL}

The definition of polytrauma has recently been updated and defined via an injury severity score of 16 and above. $^{12}$ This scoring system is based on the severity of

Figure 5 Bar chart showing

Bar chart showing anatomical and injury severity distribution anatomical distribution of of motocross injuries motocross injuries.

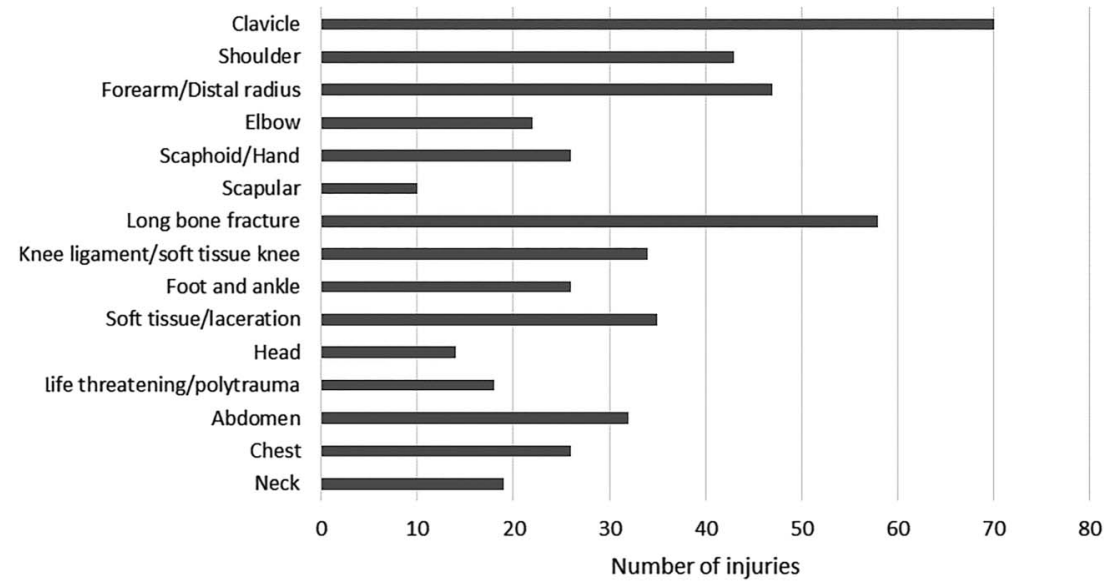




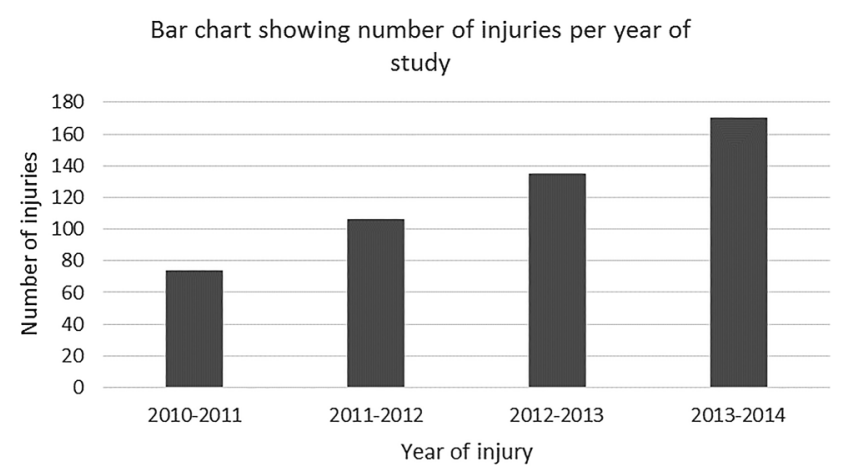

Figure 6 Bar chart showing annual increase in the number of motocross injuries.

the injury in relation to its anatomical location. We have taken this into account with our categorisation.

Off-road motocross biking often involves travelling at high speeds of up to $80 \mathrm{mph}$ down steep and hazardous

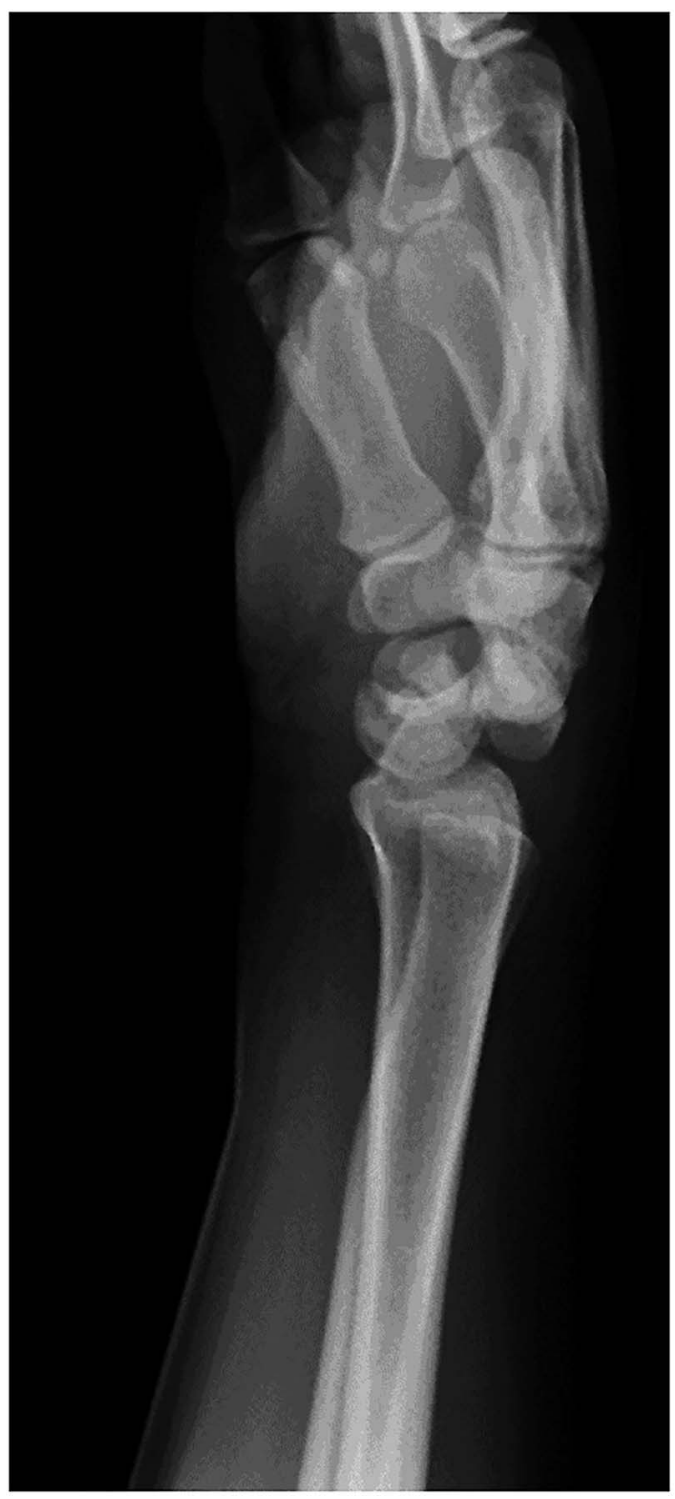

Figure 7 Lateral radiograph showing wrist carpus fracture dislocation.

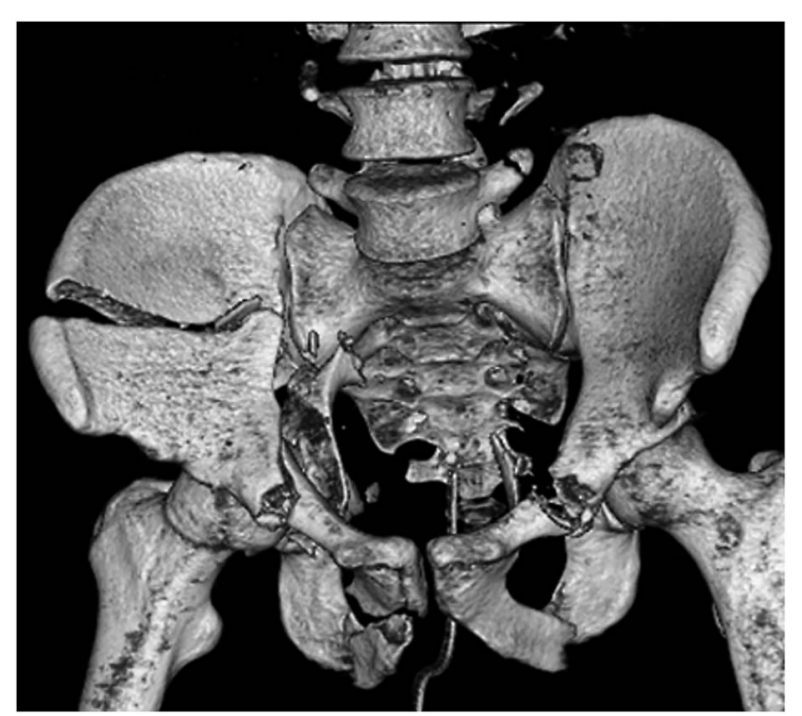

Figure 8 CT three-dimensional reconstructed coronal image showing severe bilateral pelvic fracture.

inclines with loose terrain. ${ }^{11}$ It is the sense of adventure of this uncertain and often treacherous challenge that excites the predominantly young male racer. This study shows the impact and incidence of injuries related to motocross where the collisions are high energy in nature. Indeed, several case reports of individual serious injuries caused by the use of motocross have been published. ${ }^{13-15}$ However, this is the first data series representing a collective spectrum of injuries occurring in the UK. Motocross continues to globally grow in popularity and the incidence of related injuries has grown enormously in recent years. It has been documented that

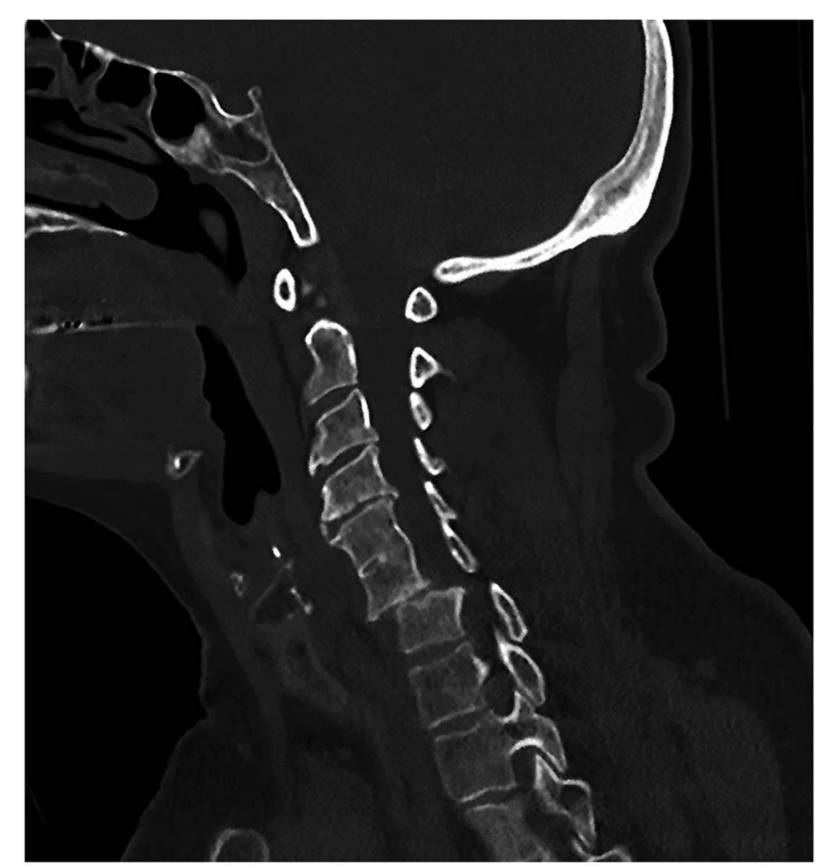

Figure 9 Sagittal CT scan showing bilateral facet cervical spine fracture dislocation. 


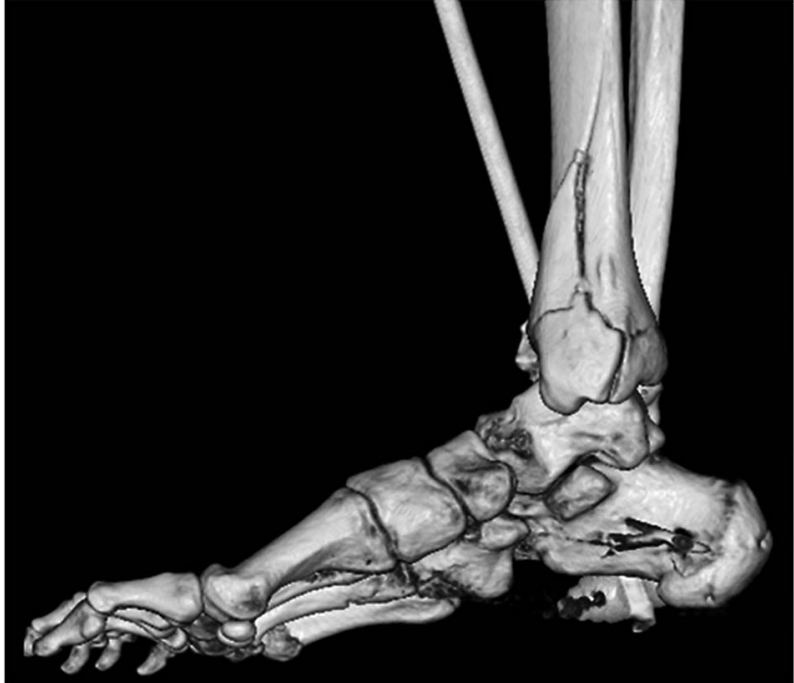

Figure 10 CT three-dimensional reconstructed coronal image showing highly comminuted intra-articular multifragmentary distal tibial fracture.

between 1997 and 2006 the overall injury rate from motocross has increased by $240 \%$, and spine injury rate by almost $500 \%$. $^{16}$

The catalogue of patients who were involved in this study was largely managed in our unit as inpatients or in our fracture clinics.

Seven patients with significant head injuries were transferred by air ambulance to specialist neurosurgical units and four patients were transferred to our neighbouring specialist spinal centre for urgent stabilisation of cervical spine fracture dislocations. Three further patients required transfer to a specialist cardiothoracic centre following life-threatening haemothorax and one for life-threatening pneumothorax.

Since this is an epidemiological study, we have not presented individual case reports; however, the severity of

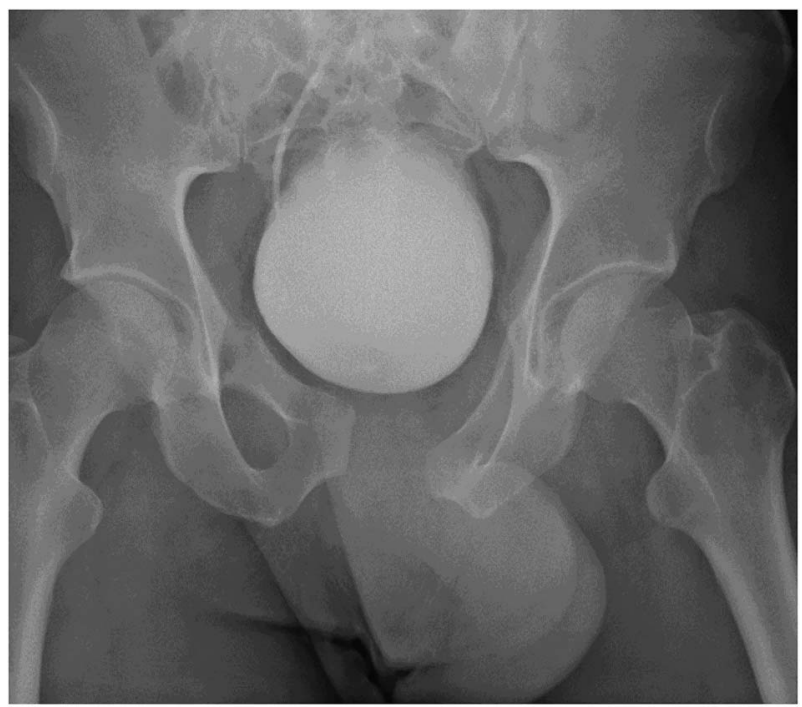

Figure 11 Plain radiograph showing unstable open-book pelvic fracture.

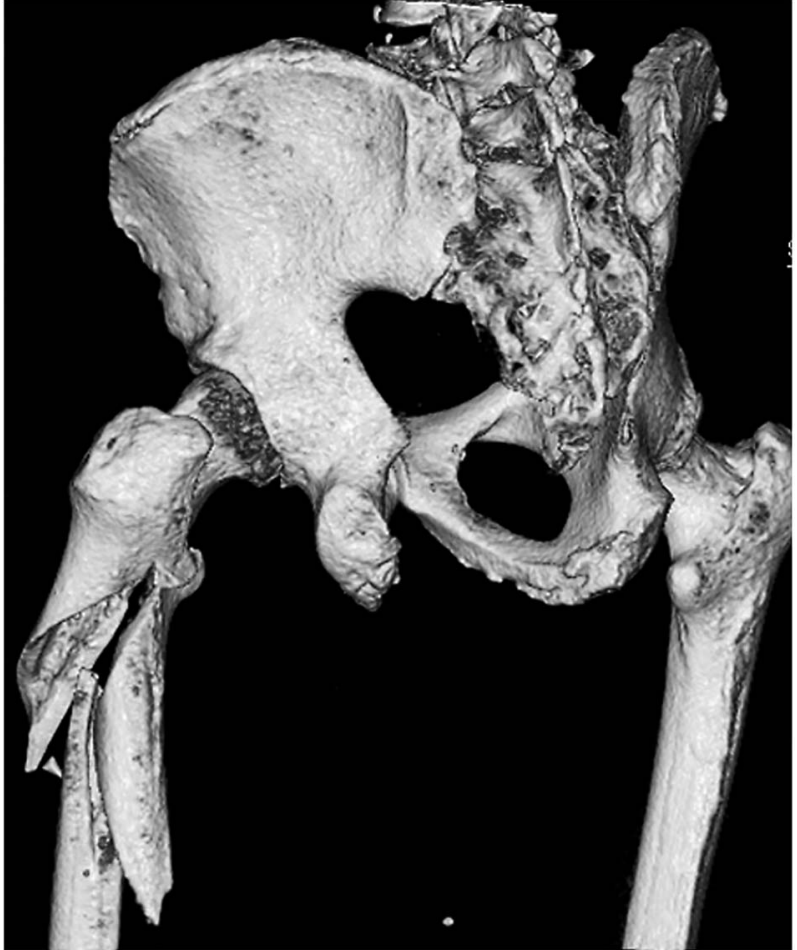

Figure 12 CT three-dimensional reconstructed coronal image showing comminuted multifragmentary proximal femur fracture.

the nature of some of the injuries as a result of motocross riding is illustrated in the figures below (figures 7-13).

A previous study in the 1980 s retrospectively collected data on motocross injuries via race medical reports, insurance declarations and follow-up forms completed by riders involved in accidents. This study stated that the overall incidence of motocross injuries was 94.5 per thousand racers, representing a risk of accident of 22.72

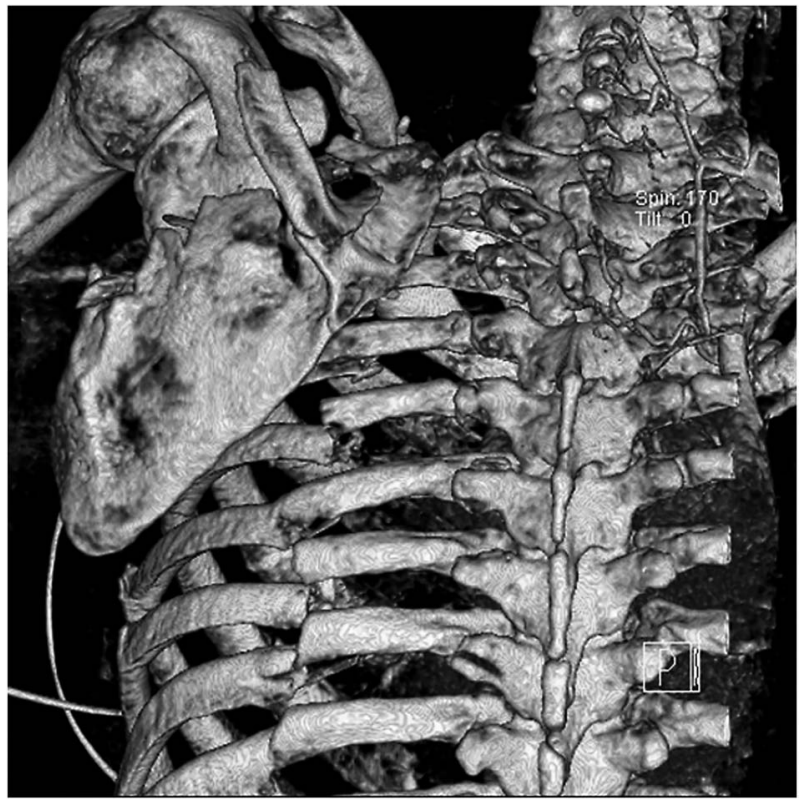

Figure 13 CT three-dimensional reconstructed coronal image showing multiple rib fractures with floating scapula. 
per thousand hours of riding. This study also found that the vast majority of fractures secondary to motocross riding were in the upper extremities $(50.9 \%) .^{10}$

\section{ECONOMIC IMPLICATION}

The estimated cost of an acute hospital bed in the UK is approximately £250 per day, and when considered in conjunction with operative intervention costs of approximately $£ 5000$ per day and follow-up fees, there is clearly a significant cost implication to the National Health Service from these injuries. ${ }^{17} 18$ In addition, many patients will be unable to work during their rehabilitation period and some may sadly suffer lasting morbidity resulting in long-term disability and care requirements.

\section{CONCLUSION}

We present the first epidemiological study of motocross injuries in the UK. Within the growing culture of 'adrenaline sports', motocross has become an exhilarating and extremely fashionable pastime. This study has identified and categorised the spectrum of associated injuries, some of which are serious and may cause significant morbidity and possible mortality. We believe that these injuries have significant resource implications, especially for smaller rural hospitals, which did not exist before the recent surge in its popularity, as illustrated by the number of injuries doubling in the past 4 years. Given the findings of the study, it is important that the receiving teams when confronted with a motocross injury take into account the mechanism and prepare for significant trauma often multiple. (Such injuries often involve high velocity impact despite the use of protective clothing).

Motocross is an attractive and mainstream sport with attendant risk of injury. Perhaps there could be an initiative for safer course design, restrictions on participant age and limitations in vehicle speeds in an aid to help reduce the incidence and the severity of injury. These eventsusually held at weekends-can also generate a sudden trauma workload often stretching local hospital facilities, at a time when resources are reduced. Understandably, this may have a knock-on effect on the treatment of other patients, both acute and elective. There is good economic and practical argument therefore to work with the event organisers, the sport's governing body (Auto Cycle Union British motocross-ACUBM) and the company's manufacturing protective equipment in an effort to improve both safety and outcome-primarily for the competitor.
Further investigations are underway to establish the prevalence and effectiveness of body armour by recreational and competitive motocross bikers, which may prove a valuable step in improving the safety of this sport. ${ }^{11}$

Contributors RS participated in the data collection and writing of the manuscript. AKH contributed to the data collection. SH contributed to the study design and project supervision.

Competing interests None declared.

Provenance and peer review Not commissioned; externally peer reviewed.

Open Access This is an Open Access article distributed in accordance with the Creative Commons Attribution Non Commercial (CC BY-NC 4.0) license, which permits others to distribute, remix, adapt, build upon this work noncommercially, and license their derivative works on different terms, provided the original work is properly cited and the use is non-commercial. See: http:// creativecommons.org/licenses/by-nc/4.0/

\section{REFERENCES}

1. Setright LJK. The Guinness book of motorcycling facts and feats. Guinness Superlatives, 1979;202, 211. ISBN: 0-85112-200-0.

2. Stealey B. History of Motocross. Retrieved 8 October 2010

3. Edison Dye and his Flying Circus. motorcyclemuseum.org. Retrieved 11 October 2011.

4. http://www.amca.uk.com/

5. At THREE years old, meet the world's youngest motocross rider, who is already competing against kids twice his size. Mail online. 16 July 2012. dailymail.co.uk.

6. Jeys L, Cribb G, Toms A, et al. Mountain biking injuries in rural England. Br J Sports Med 2001;35:197-9.

7. Carmont M. Mountain biking injuries: a review. Br Med Bull 2008;85:101-12.

8. Minoyama $\mathrm{O}$, Tsuchida $\mathrm{H}$. Injuries in professional motor car racing drivers at a racing circuit between 1996 and 2000. Br J Sports Med 2004;38:613-16.

9. Sabeti-Aschraf M, Serek M, Pachtner T, et al. Accidents and injuries in competitive Enduro motorcyclists: a prospective analysis. Knee Surg Sports Traumatol Arthrosc 2009;17:695-702.

10. Gobbi A, Tuy B, Panuncialman I. The incidence of motocross injuries: a 12-year investigation. Knee Surg Sports Traumatol Arthrosc 2004;12:574-80.

11. Grange JT, Bodnar JA, Corbett SW. Motocross medicine. Curr Sports Med Rep 2009;8:125-30.

12. Keel M, Trentz O. Pathophysiology of polytrauma. Injury 2005;36:691-709.

13. Jans C, Peersman G, Peersman B, et al. Endoscopic decompression for chronic compartment syndrome of the forearm in motocross racers. Knee Surg Sports Traumatol Arthrosc 2015;23:2522-7.

14. Gielen JL, Peersman B, Peersman G, et al. Chronic exertional compartment syndrome of the forearm in motocross racers: findings on MRI. Skeletal Radiol 2009;38:1153-61.

15. Roberts DJ, Ouellet JF, McBeth PB, et al. The "weekend warrior": fact or fiction for major trauma? Can J Surg 2014;57:E62-8.

16. Pollack P. Motocross, ATV popularity leads to rise in pediatric injuries. American Academy of Orthopaedic Surgeons.

17. Friedman $\mathrm{AB}$. Economic incentives and use of the intensive care unit. JAMA 2014;311:2336-7.

18. McLellan A. Operating theatre management. Health Ser J Health Care Leaders 2014 